\title{
Irrational rotations motivate measurable sets
}

\author{
Rodney Nillsen \\ Rodney Nillsen studied literature, mathematics and science at the University of \\ Tasmania, and proceeded to postgraduate work at the Flinders University of South \\ Australia under Igor Kluvánek. He has held academic positions at the University of \\ Malta and The University of Wales, Swansea. He is currently at the University of \\ Wollongong, New South Wales. His mathematical interests are primarily in analysis, \\ especially harmonic analysis, and he has an interest in expository mathematics. $\mathrm{He}$ \\ believes that changing social circumstances make it incumbent upon mathematicians \\ to find new ways of making the beauty and applicability of mathematical ideas \\ more accesssible to ordinary students. Outside mathematics, his interests include \\ conversation with his family, literature, classical music and the enjoyment of nature.
}

\section{Introduction}

In the general theory of integration and, in particular, measure theory, the notion of a measurable set, plays a central rôle. Various definitions of the notion of a measurable set have been used, but perhaps the most famous is the one given by Carathéodory in 1914 [2]. Carathéodory's definition depends on the notion of outer measure. Given a set $X$ an outer measure on $X$ is a function $\mu_{*}$ whose domain is the set of all subsets of $X$ and which has the following properties:

(i) $\mu_{*}(\emptyset)=0$, where $\emptyset$ denotes the empty set,

(ii) $\mu_{*}(C) \in[0, \infty]$ for all subsets $C$ of $X$, and

Mit der zunehmenden Bedeutung der Stochastik ist der Masstheorie als eines ihrer grundlegenden Werkzeuge besondere Aufmerksamkeit zu schenken. Eine solide Einführung in die Masstheorie ist auf der anderen Seite in der Studienausbildung aus Zeitgründen nicht immer einfach zu realisieren. So wird in der Regel das Riemannsche Mass und Integral vollständig zugrunde gelegt, ein ausführliches Studium der Lebesgueschen Integrationstheorie sollte aber ebenso erfolgen. Ein Zugang zur allgemeinen Mass- und Integrationstheorie wird nach Carathéodory über das sogenannte äussere Mass gegeben. Allerdings ist dieser Zugang zunächst nicht leicht zu motivieren. Im folgenden Beitrag wird der Versuch unternommen, diese Problematik anhand des Einheitskreises exemplarisch zu überwinden, indem das äussere Mass gewisser Teilmengen des Einheitskreises auf elementare Weise berechnet wird. $j k$ 
(iii) if $\left(C_{n}\right)$ is any sequence of subsets of $X$, then $\mu_{*}\left(\bigcup_{n=1}^{\infty} C_{n}\right) \leq \sum_{n=1}^{\infty} \mu_{*}\left(C_{n}\right)$.

Then, a subset $A$ of $X$ is called measurable if, for all subsets $B$ of $X$,

$$
\mu_{*}(B)=\mu_{*}(A \cap B)+\mu_{*}\left(A^{c} \cap B\right) .
$$

(Here, $A^{c}$ denotes the complement of the set $A$.) The significance of this definition lies in the fact that the set of all measurable subsets of $X$ forms a sigma algebra upon which the outer measure is countably additive. That is, if $C$ is a measurable set, and if $\left(C_{n}\right)$ is a sequence of measurable sets, then the complement of $C$ is measurable and $\bigcup_{n=1}^{\infty} C_{n}$ is measurable; and if furthermore the sets in $\left(C_{n}\right)$ are pairwise disjoint then $\mu_{*}\left(\bigcup_{n=1}^{\infty} C_{n}\right)=\sum_{n=1}^{\infty} \mu_{*}\left(C_{n}\right)$. These properties produce an integration theory which allows for the natural interchange of limits and integrals (Lebesgue's dominated convergence theorem), and the interchange of the order of integration in multiple integrals (Fubini's theorem).

Since Carathéodory originally gave his definition of a measurable set, it has frequently been the subject of comment or defence in a way unusual for the definition of a mathematical concept. For example, in their Real and Abstract Analysis [6, p. 127], Edwin Hewitt and Karl Stromberg write: "How Carathéodory came to think of this definition seems mysterious, since it is not in the least intuitive. Carathéodory's definition has many useful implications". Also, Paul Halmos comments in his Measure Theory [4, p. 44]: "It is rather difficult to get an understanding of the meaning of ... measurability except through familiarity with its implications... The greatest justification of this apparently complicated concept is, however, its possibly surprising but absolutely complete success as a tool in proving the important and useful extension theorem". However, not everyone has accepted that the definition of a measurable set can be justified by appealing to a usefulness which may be at the time quite unclear but which will be amply justified in the future. Writing in his Proofs and Refutations [7, pp. 153-154] about Michel Loève's treatment [8, p. 87] of measurable sets, Imre Lakatos says: “... how on earth can he know which of these most complicated instruments will be needed for the operation? Certainly he already has some idea what he will find and how he will proceed. But why then, this mystical set-up of putting the definition before the proof?" He goes on to write: "... stating the primitive conjecture, showing the [attempted] proof, the counter examples, and following the heuristic order up to the theorem and to the proof-generated definition would dispel the authoritarian mysticism of abstract mathematics and would act as a brake on degeneration."

In his objections, Lakatos is essentially arguing that pedagogical transparency should have priority over what he considers to be purely intellectual efficiency; and that placing the definition before the point at which the reason for the definition becomes clear has an authoritarian aspect to it which the serious teacher should reject. The case is argued forcibly in [7, pp. 127-154]. Lakatos distinguishes between the "deductivist" approach and his preferred "heuristic" approach. However, the "deductivist" approach 
is not inherently authoritarian. After all, the purpose of a proof is to give a reasoned argument for mathematical assertions, and this argument must be more than the mere opinions of the teacher. Although it may be the teacher who draws explicit attention to the reasoning in the argument, he or she is subject to that reasoning as much as is the student, so that the student may draw attention to errors. It is when the student does not humanly engage with the proof, or grasp its purpose, that the deductivist method becomes authoritarian. After all, if we listen to Mozart's fortieth symphony, do we question at each note why Mozart used this or that note rather than another? No, instead, we give ourselves over to the music. At its best, it is this type of experience which mathematical proof gives. From all these viewpoints, the danger of intellectual authoritarianism lies with the possible attitudes of the teacher and the student rather than within the deductivist approach itself. However, none of this is to say that the deductivist approach invariably should be preferred, nor is it to say that there is a necessary and exclusive choice between the deductivist and heuristic approaches.

Of course, the objections of Lakatos apply not simply to the definition of a measurable set, but also to the way in which the definitions of many mathematical concepts often are presented. However, in the case of measurable sets, the situation is more acute than in many other cases. The definition of a measurable set in itself is not really counter-intuitive, since the "counter-intuitive" definition can in fact be given an intuitive interpretation, as Halmos [4, p. 44] explains. However, the definition is counter-intuitive in the sense that it seems to appear out of nowhere and its immediate intuitive interpretation does not provide a broader context, or even the suggestion of one, in which it might appear as natural, transparent or necessary. The definition of a measurable set appears to be unusually "remote" from the context which gives it its mathematical importance. On the one hand, this very "remoteness" may have an intriguing and mysterious quality. Indeed, it can be held that crucial mathematical concepts and definitions retain an intrinsic air of unexpectedness and mystery which no amount of heuristic justification or tracing of historical origins can eliminate. A good definition remains primarily an artistic act. However, on the other hand, the definition of a measurable set may have a seeming artificiality and a lack of any intrinsic indication as to its possible longer-term significance.

The pedagogical questions raised by Lakatos' objections to the usual presentations of the definition of a measurable set are complex, and it is not the intention to pursue them further here (a discussion of Lakatos and his ideas may be found in the book of Reuben Hersh [5], especially pp. 208-216). Rather, the present paper arose from the fact that many applications of analysis assume a prior knowledge of measure theory, a situation which creates a barrier for many undergraduate students, who are thereby prevented by seeing some of the most interesting applications of modern analysis. In this paper, we consider the problem of calculating the outer measure of an invariant set of an irrational rotation on the circle group, without assuming prior knowledge of measure theory or the concept of a measurable set. It is shown that if the invariant set satisfies a certain condition, then the outer measure of the set is either 0 or 1 . This "certain condition" arises naturally from the problem, rather than being artifically imposed, and it takes a form which is very similar to Carathéodory's definition of a measurable set. 
So, although the motivation for it was different, the work here can be considered to show how Carathéodory's definition of a measurable set arises from a specific "problem situation", a pedagogical approach recommended by Lakatos.

\section{The ergodicity problem on the unit circle}

The unit circle $\mathbb{T}$ is the set of all complex numbers of modulus one. The set $\mathbb{T}$ is a group under multiplication of complex numbers, and for this reason $\mathbb{T}$ is also called the circle group. A rotation on $\mathbb{T}$ is a function $\rho: \mathbb{T} \longrightarrow \mathbb{T}$ for which there exists $z \in \mathbb{T}$ such that

$$
\rho(w)=z w, \quad \text { for all } \quad w \in \mathbb{T} .
$$

That is, a rotation on $\mathbb{T}$ is a function from $\mathbb{T}$ to $\mathbb{T}$ which is given by multiplication by some fixed element of $\mathbb{T}$. A group theoretic viewpoint would be that a rotation in $\mathbb{T}$ is a translation in the group $\mathbb{T}$. Geometrically, a rotation given by (2.1) will rotate points anti-clockwise through an angle $2 \pi \alpha$, where $\exp (2 \pi i \alpha)=z$. If $\alpha$ is irrational, the rotation is called irrational, otherwise the rotation is rational. Alternatively, a rotation given by (2.1) is irrational if $z$ is not a root of unity. Given a rotation $\rho$, a subset $A$ of $\mathbb{T}$ is said to be invariant under $\rho$, or simply $\rho$-invariant if $\rho(A)=A$. That is, $A$ is invariant means that $A$ is unchanged under the action of $\rho$. For example, for each $w \in \mathbb{T},\left\{\ldots, \rho^{-2}(w), \rho^{-1}(w), w, \rho(w), \rho^{2}(w), \ldots\right\}$ is a proper $\rho$-invariant subset of $\mathbb{T}$, but it is rather "small" as a subset of $\mathbb{T}$. On the other hand, the complement of $\left\{\ldots, \rho^{-2}(w), \rho^{-1}(w), w, \rho(w), \rho^{2}(w), \ldots\right\}$, which is also invariant, is rather "large" as a subset of $\mathbb{T}$. The ergodicity problem on the unit circle may be taken to be: for a given rotation $\rho$ on $\mathbb{T}$, are there any $\rho$-invariant subsets of $\mathbb{T}$ which are neither "small" nor "large"? The precise meaning of "small" and "large" in this context is usually expressed in terms of measurable sets and their measure. Thus, it is a well-known result that if $\rho$ is an irrational rotation and $A$ is a measurable and $\rho$-invariant subset of the unit circle then either $A$ has measure 0 or $A$ has the greatest possible measure, namely the measure of the whole unit circle. That is, in the terminology of [13, p. 27] (for example), an irrational rotation on the unit circle is ergodic.

There are two main approaches for proving that an irrational rotation $\rho$ is ergodic, and both assume a background in measure theory. The first uses functional and Fourier analysis techniques in the space $L^{2}(\mathbb{T})$ to show that if $\chi_{A}$ denotes the characteristic function of a measurable $\rho$-invariant set $A$, then the Fourier coefficients of $\chi_{A}$ are all zero, except for the one corresponding to the constant term in the Fourier series. It follows that $\chi_{A}$ is constant, up to a set of measure zero, but also $\chi_{A}$ assumes only the values 0 and 1 . It follows that either $\chi_{A}$ is 0 everywhere except for a set of measure zero, in which case $A$ is "small"; or $\chi_{A}$ is 1 everywhere except for a set of measure zero, in which case $A$ is "large". This approach may be found in [13, pp. 29-30], for example.

The second approach for proving that an irrational rotation $\rho$ is ergodic is based upon the measure-theoretic notion of points of density. A point $x$ is a point of density for a set $A$ if "most" of the points near $x$ belong to $A$ (see [6, p. 274] or [12, p. 177] for details of this concept). Now in [12, pp. 39-40], Y.G. Sinai assumes that $B, C$ are disjoint measurable 
$\rho$-invariant sets of strictly positive measure, and considers points of density $b$ of $B$ and $c$ of $C$. Then each iterate of the rotation carries $b$ into a point which is also a point of density of $B$, but simultaneously there is at least one iterate of $\rho$, say $\rho^{[n]}$, which carries $b$ into a given neighbourhood of $c$. We deduce that $\rho^{[n]}(b)$ is simultaneously a point of density of $B$ and an "approximate" point of density of $C$. This leads to the conclusion that $B, C$ are not disjoint, a contradiction which establishes that $B$ either has measure 0 or has full measure in $\mathbb{T}$. That is, the irrational rotation is ergodic.

Of these two approaches for proving that an irrational rotation is ergodic, the first assumes a knowledge of measure and integration, complete orthonormal sets and Fourier expansions in $L^{2}$-spaces; while the second presupposes a knowledge of measure theory at a level of refinement which is not generally attained in an undergraduate course (in the book of Robert Bartle [1], for example, points of density are not discussed).

Note that just the above statement of the result that an irrational rotation is ergodic has assumed a knowledge of the notions of measurable set and the measure of a measurable set. All formulations and proofs of this result known to the author are based upon this assumed prior knowledge of measure theory.

The approach in the present paper is to consider the problem of finding the outer measure of an invariant set of an irrational rotation on $\mathbb{T}$. By considering only the outer measure, we avoid the need to consider the notion of measurable set, at least initially. Rather, we are led to the notion of measurable set by the problem of trying to say something about the outer measures of the invariant sets of an irrational rotation. However, the extent to which this satisfactorily motivates the definition of a measurable set, and overcomes objections to the definition such as made by Lakatos, must remain a judgment for the reader. From the author's viewpoint, the ideas here arose primarily from the pedagogical problem of how to motivate and present some of the ideas of ergodic theory to a class with no background in measure theory or functional analysis [9].

\section{Outer measure, rotations and invariant sets}

The function $t \longmapsto \exp (2 \pi i t)$ is one-to-one and maps $[0,1)$ onto $\mathbb{T}$, so this function enables us to think of $\mathbb{T}$ as the unit interval, an identification which is sometimes useful. We define a subinterval $J$ of $[0,1)$ to be half open if there are $b, c \in[0,1)$ with $b \leq c$ such that $J=[b, c)$. Then, for present purposes, a subset $V$ of $\mathbb{T}$ is called an arc either if there is a half open subinterval $J$ of $[0,1)$ such that

$$
V=\{\exp (2 \pi i t): t \in J\}
$$

or if there are disjoint half open subintervals $K, L$ of $[0,1)$ such that $K$ is of the form $[0, b), L$ is of the form $[c, 1)$, and

$$
V=\{\exp (2 \pi i t): t \in K \cup L\}
$$

Note that this rather cumbersome latter part of the definition is to allow for arcs which may have 1 as an interior point. If $V$ is an arc and $J$ is a subinterval of $[0,1)$ such that 
$V=\{\exp (2 \pi i t): t \in J\}$, we define the length $\mu(V)$ of $V$ to be the usual length of the interval $J$. If $V$ is an arc and $K, L$ are subintervals of $[0,1)$ such that $V=\{\exp (2 \pi i t)$ : $t \in K \cup L\}$, as above, we define the length $\mu(V)$ of $V$ to be the sum of the usual lengths of the intervals $K, L$. This definition means that the circumference of the unit circle is assigned length 1 , rather than the usual value $2 \pi$, but we work with the given definition of length for convenience.

If an arc $V$ is the disjoint union of $\operatorname{arcs} V_{1}, V_{2}, \ldots, V_{n}$, then it is easy to check that $\mu(V)=\sum_{j=1}^{n} \mu\left(V_{j}\right)$. This enables us to make the definition that if $A$ is any subset of $\mathbb{T}$ which is a finite union of arcs $V_{1}, V_{2}, \ldots, V_{n}$, then $\mu(V)$ is by definition the sum $\sum_{j=1}^{n} \mu\left(V_{j}\right)$. (Although $A$ may be expressed in different ways as a union of disjoint arcs, this sum always has the same value.)

Now, for any subset $A$ of $\mathbb{T}$, the outer measure $\mu_{*}(A)$ of $A$ is defined as follows:

$$
\mu_{*}(A)=\inf \left\{\sum_{n=1}^{\infty} \mu\left(V_{n}\right):\left(V_{n}\right) \text { is a sequence of } \operatorname{arcs} \text { in } \mathbb{T} \text { such that } A \subseteq \bigcup_{n=1}^{\infty} V_{n}\right\} .
$$

It is not difficult to prove (see [1, p. 99]) from the definition that for any sequence $\left(A_{n}\right)$ of subsets of $\mathbb{T}$,

$$
\mu_{*}\left(\bigcup_{n=1}^{\infty} A_{n}\right) \leq \sum_{n=1}^{\infty} \mu_{*}\left(A_{n}\right) .
$$

Incidentally, note that if $A$ is any subset of $\mathbb{T}$ with $\mu_{*}(A)=0$, then $A$ is measurable. For, in this case, for any subset $B$ of $\mathbb{T}$ we will have

$$
\begin{aligned}
\mu_{*}(B) & =\mu_{*}\left(B \cap\left(A \cup A^{c}\right)\right) \\
& =\mu_{*}\left((B \cap A) \cup\left(B \cap A^{c}\right)\right) \\
& \leq \mu_{*}(B \cap A)+\mu_{*}\left(B \cap A^{c}\right) \\
& \leq \mu_{*}(A)+\mu_{*}(B) \\
& =0+\mu_{*}(B) \\
& =\mu_{*}(B),
\end{aligned}
$$

so that

$$
\mu_{*}(B)=\mu_{*}(B \cap A)+\mu_{*}\left(B \cap A^{c}\right) .
$$

If $z \in \mathbb{T}$, the rotation given by (2.1) is denoted by $\rho_{z}$. Thus, $\rho_{z}(w)=z w$, for all $w, z \in \mathbb{T}$. Note that $\rho_{z}$ is the identity transformation on $\mathbb{T}$ if and only if $z=1$. If $w, z \in \mathbb{T}$ are such that $w=\exp (2 \pi i \alpha)$ and $z=\exp (2 \pi i \theta)$, then

$$
\rho_{z}(w)=z w=\exp (2 \pi i \theta) \cdot \exp (2 \pi i \alpha)=\exp (2 \pi i(\alpha+\theta))
$$

which shows formally that $\rho_{z}$ acts upon $\mathbb{T}$ by rotation through the angle $2 \pi \theta$, thus justifying the use of the term "rotation" for a function of the form $\rho_{z}$. 
Now, let $f \circ g$ denote the usual composition of the functions $f, g$. When $f$ is a function which maps a set into itself, and when $n \in \mathbb{N}$, let $f^{[n]}$ denote the composition $f \circ \cdots \circ f$, where $f$ appears $n$ times. Then, if $x, y, z \in \mathbb{T}$,

$$
\left(\rho_{y} \circ \rho_{z}\right)(x)=\rho_{y}\left(\rho_{z}(x)\right)=\rho_{y}(z x)=y z x=(y z) x=\rho_{y z}(x)
$$

so that

$$
\rho_{y} \circ \rho_{z}=\rho_{y z}, \quad \text { for all } y, z \in \mathbb{T} \text {. }
$$

When a function $f$ has an inverse $f^{-1}, f^{[-n]}$ denotes $\left(f^{-1}\right)^{[n]}$. A rotation $\rho$ is one-to-one and onto, so it has an inverse. It follows that if $\mathbb{Z}$ denotes the set of integers,

$$
\rho_{z}^{[n]}=\rho_{z^{n}}, \text { for all } z \in \mathbb{T} \text { and all } n \in \mathbb{Z}
$$

If $\rho$ is a rotation, $\rho(J)$ is an arc whenever $J$ is an arc, and in this case $\mu(\rho(J))=\mu(J)$. It follows from this observation and (3.1) that

$$
\mu_{*}(\rho(A))=\mu_{*}(A)
$$

for all subsets $A$ of $\mathbb{T}$.

Now, if $\rho$ is a rotation on $\mathbb{T}$, and if $A$ is a subset of $\mathbb{T}$, we say that $A$ is $\rho$-invariant when $\rho(A)=A$. The empty set is $\rho$-invariant and so is the set $\mathbb{T}$. When $\rho=\rho_{z}, A$ is $\rho$-invariant precisely when $z A=A$. The following result characterizes completely the invariant sets of a rational rotation, and is worth thinking about purely geometrically.

Proposition 3.1 Let $\alpha=p / q$ be a rational number in $[0,1)$, where $p, q \in \mathbb{N}$ and have no common factors. Let $z=\exp (2 \pi i p / q)$, and let $A$ be a subset of $\mathbb{T}$. Then the following conditions are equivalent:

(1) There is a subset $B$ of the arc $\{\exp (2 \pi i t): 0 \leq t<1 / q\}$ such that $A=\bigcup_{j=1}^{q} z^{j-1} B$.

(2) A is $\rho_{z}$-invariant.

Proof. Let (1) hold. Observe that $z^{q}=(\exp (2 \pi i p / q))^{q}=\exp (2 \pi i)=1$. We now have

$$
\rho_{z}(A)=z A=z\left(\bigcup_{j=1}^{q} z^{j-1} B\right)=\bigcup_{j=1}^{q} z^{j} B=\left(\bigcup_{j=1}^{q-1} z^{j} B\right) \cup B=\bigcup_{j=1}^{q} z^{j-1} B=A,
$$

so that $A$ is $\rho_{z}$-invariant and (2) holds. Conversely, let (2) hold and for $j=1,2, \ldots, q$ put

$$
J_{j}=\left\{\exp (2 \pi i t): \frac{j-1}{q} \leq t<\frac{j}{q}\right\}
$$

and also put

$$
B=A \cap\left\{\exp (2 \pi i t): 0 \leq t<\frac{1}{q}\right\}
$$


Note that $\mathbb{T}=\bigcup_{j=1}^{q} J_{j}$ and that $B=A \cap J_{1}$. Now as $A$ is $\rho_{z}$-invariant,

$$
\bigcup_{j=1}^{q} z^{j-1} B \subseteq \bigcup_{j=1}^{q} z^{j-1} A=\bigcup_{j=1}^{q} A=A
$$

On the other hand, let $x \in A$. Then, as $\mathbb{T}=\bigcup_{j=1}^{q} J_{j}$, we have $x \in J_{j}$ for some $j \in$ $\{1,2, \ldots, q\}$, so that $x=\exp (2 \pi i t)$ for some $t \in[(j-1) / q, j / q)$. Let $z_{0}=\exp (2 \pi i / q)$. Also, note that as $p, q$ have no common factors, every $q^{\text {th }}$ root of unity is of the form $z^{k}$ for some $k \in\{0,1,2, \ldots, q-1\}$. As $z_{0}^{j-1}$ is a $q^{\text {th }}$ root of unity, there is $\ell \in\{1,2, \ldots, q\}$ such that $z_{0}^{j-1}=z^{\ell-1}$. We now have

$$
z^{-\ell+1} x=z_{0}^{-j+1} x=\exp (-2 \pi i(j-1) / q) \exp (2 \pi i t)=\exp (2 \pi i(t-(j-1) / q)) \in J_{1} \text {. }
$$

But as $x \in A$ and as $A$ is $\rho_{z}$-invariant, $z A=A$ and $z^{-\ell+1} A=A$, which gives $z^{-\ell+1} x \in A$. Thus, $z^{-\ell+1} x \in A \cap J_{1}=B$. It now follows that $x \in z^{\ell-1} B$, so that

$$
A \subseteq \bigcup_{j=1}^{q} z^{j-1} B
$$

As we have now seen that $\bigcup_{j=1}^{q} z^{j-1} B \subseteq A$ and $A \subseteq \bigcup_{j=1}^{q} z^{j-1} B$, it follows that

$$
A=\bigcup_{j=1}^{q} z^{j-1} B
$$

Because $B \subseteq J_{1}$, this shows that (2) implies (1).

Proposition 3.1 characterizes the invariant sets of a rational rotation, but what about irrational rotations? Given a rational rotation $\rho_{z}$, as in Proposition 3.1, we see that its invariant sets may be constructed from any subset $B$ of the $\operatorname{arc}\{\exp (2 \pi i t): 0 \leq t<$ $1 / q\}$, by taking the union of $B$ with $q-1$ copies of $B$, each of which is obtained by a rotation by some iterate of $\rho_{z}$. Note that because the $\operatorname{arc}\{\exp (2 \pi i t): 0 \leq t<1 / q\}$ has length $1 / q$, the subset $B$ must have outer measure at most $1 / q$. Now, every irrational number is the limit of a sequence $\left(p_{n} / q_{n}\right)$ of rational numbers with $p_{n}, q_{n} \in \mathbb{N}$ and $\lim _{n \rightarrow \infty} q_{n}=\infty$. Because $\lim _{n \rightarrow \infty} 1 / q_{n}=0$, by analogy with the rational rotations, we might anticipate that any "non-trivial" proper subset of $\mathbb{T}$ which is an invariant set of some irrational rotation will be found by taking an infinite but countable number of copies of some set of outer measure zero. On the other hand, $\mathbb{T}$ is an invariant set whose complement has outer measure zero, and there may be other subsets of $\mathbb{T}$ which are also invariant and have outer measure zero. So, we might think that the following conjecture is reasonable and that a result along these lines would complement the result for rational rotations in Proposition 3.1.

Conjecture. Let $A$ be a subset of $\mathbb{T}$ which is an invariant set for some irrational rotation $\rho$. Then either $\mu_{*}(A)=0$ or $\mu_{*}\left(A^{c}\right)=0$.

The aim is to investigate this conjecture with the only measure theoretic tool being the outer measure. 


\section{Arcs of density}

Suppose a subset of $\mathbb{T}$ has positive outer measure. Then what can be said about that set? In this section we show that such a set has a part consisting of points which "mostly" belong to an arc, and such an arc might loosely be termed an "arc of density" of the set. Alternatively, we might think of such an arc as having "most" of its points in the set. Since the outer measure is obtained by approximating the given set by sequences of arcs, reflection upon equation (3.1) will make the existence of arcs of density seem to be not too surprising. There is a resemblance between the concepts of an "arc of density" and a "point of density", but the existence of the former can be established naturally and easily within the context of outer measure only. Once we know that arcs of density exist, it is shown that these arcs may be chosen to have any given length, provided that this length is sufficiently small relative to the set which is assumed to have positive outer measure. The significance of these technicalities lies in the fact that if $A$ is an invariant set for some irrational rotation such that both $A$ and $A^{c}$ have positive outer measure, then both $A$ and $A^{c}$ will have arcs of density of equal length. Then, some iterate of the irrational rotation will approximately rotate one of these arcs into the other and, depending on the nature of the set $A$, this may lead to the contradictory conclusion that $A$ and $A^{c}$ have points in common!

The following result establishes the existence of arcs of density for sets of positive outer measure.

Lemma 4.1 Let $A \subseteq \mathbb{T}$, let $\mu_{*}(A)>0$ and let $\varepsilon>0$. Then there is a non-empty arc $J$ of $\mathbb{T}$ such that

$$
\frac{\mu_{*}(A \cap J)}{\mu(J)}>1-\varepsilon
$$

Proof. Assume that the result is false. Then, for any non-void $\operatorname{arc} W$ of $\mathbb{T}$,

$$
\mu_{*}(A \cap W) \leq(1-\varepsilon) \mu(W) .
$$

Now let $\delta>0$. By the definition of $\mu_{*}(A)$ there is a sequence $\left(W_{n}\right)$ of non-void arcs of $\mathbb{T}$ such that

$$
A \subseteq \bigcup_{n=1}^{\infty} W_{n} \text { and } \sum_{n=1}^{\infty} \mu\left(W_{n}\right)<\mu_{*}(A)+\delta
$$

Then $A \subseteq \bigcup_{n=1}^{\infty}\left(A \cap W_{n}\right)$, so if we use (3.2) and (4.1), it follows that

$$
\mu_{*}(A) \leq \sum_{n=1}^{\infty} \mu_{*}\left(A \cap W_{n}\right) \leq(1-\varepsilon) \sum_{n=1}^{\infty} \mu\left(W_{n}\right) \leq(1-\varepsilon)\left(\mu_{*}(A)+\delta\right)
$$

Hence

$$
\mu_{*}(A) \leq \frac{\delta(1-\varepsilon)}{\varepsilon}
$$


and this is true for all $\delta>0$. Hence, if the result is false, $\mu_{*}(A)=0$, which contradicts the assumed fact that $\mu_{*}(A)>0$.

The following result is a refinement of the preceding one. Whereas the preceding result says that a given set of positive measure has a part which is "approximately" an arc, we can think of this new result as saying that this part of the set may be chosen to be "small", in the sense that it is "approximately" an arc which can have any given length, as long as that length is sufficiently small.

Lemma 4.2 Let $A \subseteq \mathbb{T}$, let $\mu_{*}(A)>0$ and let $\varepsilon>0$. Then there is a number $\theta>0$ which has the following property: if $\eta \in(0, \theta)$, there is an arc $J$ of $\mathbb{T}$ such that

$$
\mu(J)=\eta \quad \text { and } \quad \frac{\mu_{*}(A \cap J)}{\mu(J)}>1-\varepsilon .
$$

Proof. If $\varepsilon>1,1-\varepsilon<0$, in which case the result is obviously true. So it may be assumed that $\varepsilon \leq 1$. By Lemma 4.1, there is a non-empty arc $J^{\prime}$ of $\mathbb{T}$ such that

$$
\frac{\mu_{*}\left(A \cap J^{\prime}\right)}{\mu\left(J^{\prime}\right)}>1-\frac{\varepsilon}{2}
$$

Let us define

$$
\theta=\frac{\mu\left(J^{\prime}\right)}{2}
$$

so that

$$
2 \theta=\mu\left(J^{\prime}\right)>0 .
$$

Now let $\eta$ be any positive number with $\eta<\theta$. Then

$$
0<\eta<\theta<2 \theta=\mu\left(J^{\prime}\right) .
$$

Hence there are $m \in \mathbb{N}$ and $\eta^{\prime} \in[0, \eta)$ such that

$$
\mu\left(J^{\prime}\right)=m \eta+\eta^{\prime}
$$

Then there are $m+1$ consecutive disjoint arcs $Z_{1}, Z_{2}, \ldots, Z_{m}, Z$ of $\mathbb{T}$ such that

$$
\mu\left(Z_{1}\right)=\eta, \mu\left(Z_{2}\right)=\eta, \ldots, \mu\left(Z_{m}\right)=\eta, \mu(Z)=\eta^{\prime}, \text { and } J^{\prime}=\left(\bigcup_{j=1}^{m} Z_{j}\right) \cup Z
$$


Now assume that $\mu_{*}\left(A \cap Z_{j}\right) \leq(1-\varepsilon) \eta$ for all $j=1,2, \ldots, m$. Then, by (4.2),

$$
\begin{aligned}
\left(1-\frac{\varepsilon}{2}\right) \mu\left(J^{\prime}\right) & <\mu_{*}\left(A \cap J^{\prime}\right) \\
& =\mu_{*}\left(A \cap\left[\left(\bigcup_{j=1}^{m} Z_{j}\right) \cup Z\right]\right) \\
& =\mu_{*}\left(\left(\bigcup_{j=1}^{m} A \cap Z_{j}\right) \cup A \cap Z\right) \\
& \leq\left(\sum_{j=1}^{m} \mu_{*}\left(A \cap Z_{j}\right)\right)+\mu_{*}(A \cap Z) \\
& \leq\left(\sum_{j=1}^{m}(1-\varepsilon) \eta\right)+\mu(Z) \\
& =(1-\varepsilon)\left(\left(\sum_{j=1}^{m} \eta\right)+\mu(Z)\right)+\varepsilon \mu(Z) \\
& =(1-\varepsilon)\left(\left(\sum_{j=1}^{m} \mu\left(Z_{j}\right)\right)+\mu(Z)\right)+\varepsilon \mu(Z) \\
& =(1-\varepsilon) \mu\left(\left(\bigcup_{j=1}^{m} Z_{j}\right) \cup Z\right)+\varepsilon \mu(Z) \\
& =(1-\varepsilon) \mu\left(J^{\prime}\right)+\varepsilon \mu(Z)
\end{aligned}
$$

by (4.4). Using (4.3) and (4.4), we see that this implies that

$$
\mu\left(J^{\prime}\right)<2 \mu(Z)=2 \eta^{\prime}<2 \eta<2 \theta=\mu\left(J^{\prime}\right),
$$

which is a contradiction. Hence, the assumption that $\mu_{*}\left(A \cap Z_{j}\right) \leq(1-\varepsilon) \eta$ for all $j=1,2, \ldots, m$ is wrong, and it follows that $\mu_{*}\left(A \cap Z_{j}\right)>(1-\varepsilon) \eta=(1-\varepsilon) \mu\left(Z_{j}\right)$ for some $j \in\{1,2, \ldots, m\}$. If $Z_{j}$ is an arc with this property, and if we put $J=Z_{j}$, then

$$
\mu(J)=\eta \quad \text { and } \quad \frac{\mu_{*}(A \cap J)}{\mu(J)}>(1-\varepsilon),
$$

as required.

If $A, B$ are sets, let $A \triangle B$ denote the set given by

$$
A \triangle B=\left(A \cap B^{c}\right) \cup\left(A^{c} \cap B\right) .
$$


Note that $A=B$ if and only if $A \triangle B=\emptyset$. Now, when $A, B$ are also subsets of $\mathbb{T}$, we have

$$
\mu_{*}(A)=\mu_{*}((A \cap B) \cup(A \triangle B)) \leq \mu_{*}(B \cup A \triangle B) \leq \mu_{*}(B)+\mu_{*}(A \triangle B),
$$

so that

$$
\mu_{*}(A)-\mu_{*}(B) \leq \mu_{*}(A \triangle B) .
$$

As this inequality is symmetric in $A, B$ we have also

$$
\mu_{*}(B)-\mu_{*}(A) \leq \mu_{*}(A \triangle B) .
$$

It follows that for any subsets $A, B$ of $\mathbb{T}$,

$$
\left|\mu_{*}(B)-\mu_{*}(A)\right| \leq \mu_{*}(A \triangle B),
$$

a fact we use in the following lemma.

Lemma 4.3 Let $z$ be an element of $\mathbb{T}$ which is not a root of unity, let $J, K$ be non-empty arcs in $\mathbb{T}$ which are of equal length, and let $\varepsilon>0$. Then there is some $n \in \mathbb{N}$ such that

$$
\mu\left(z^{n} J \triangle K\right)<\varepsilon .
$$

Proof. As $J, K$ have equal length, there is $w \in \mathbb{T}$ such that $w J=K$. Also, there is an $\operatorname{arc} V$ containing 1 such that

$$
\mu(y J \triangle J)<\varepsilon \text { for all } y \in V .
$$

Now as seen from $\left[4\right.$, p. 69], the set $\left\{z^{n}: n \in \mathbb{N}\right\}$ is dense in $\mathbb{T}$, so that there is $n \in \mathbb{N}$ such that $z^{n} \in w V$. That is, $z^{n} w^{-1} \in V$.

So, we now have, using (3.4) and (4.6):

$$
\begin{aligned}
\mu\left(z^{n} J \triangle K\right) & =\mu\left(z^{n} J \triangle w J\right) \\
& =\mu\left(w^{-1}\left(z^{n} J \triangle w J\right)\right) \\
& =\mu\left(z^{n} w^{-1} J \triangle J\right) \\
& <\varepsilon,
\end{aligned}
$$

as required.

The conclusion of Lemma 4.3 may seem more obvious if it is thought of in purely geometric terms. For, if we have two arcs of equal length, there is a rotation which exactly rotates one arc into the other - but this rotation may be approximated by some iterate of the given irrational rotation, so that such an iterate will "approximately" rotate one arc into the other. 


\section{Measurability materializes}

In this section we come to grips with the problem of saying something about the outer measure of the invariant sets of an irrational rotation on $\mathbb{T}$. The main result we obtain motivates the formal definition of a measurable set. The technical preliminaries have been developed in Section 4, and we now put them into action.

Let $z$ be an element of $\mathbb{T}$ which is not a root of unity, and let $A$ be an invariant set of the irrational rotation $\rho_{z}$ such that $\mu_{*}(A)>0$ and $\mu_{*}\left(A^{c}\right)>0$. We investigate the consequences of this assumption. To this end, let $\varepsilon$ be any number with $\varepsilon>0$. Then, by Lemma 4.2, there are non-empty arcs $J, K$ of $\mathbb{T}$ such that

$$
\mu(J)=\mu(K), \quad \frac{\mu_{*}(A \cap J)}{\mu(J)}>1-\varepsilon, \quad \text { and } \quad \frac{\mu_{*}\left(A^{c} \cap K\right)}{\mu(K)}>1-\varepsilon .
$$

Now let $\delta>0$. As $z$ is not a root of unity, $z^{-1}$ is also not a root of unity. So by Lemma 4.3 there is $n \in \mathbb{N}$ such that

$$
\mu\left(z^{-n} J \triangle K\right)<\delta .
$$

Now observe that

$$
A^{c} \cap K \subseteq\left(A^{c} \cap z^{-n} J\right) \cup\left(z^{-n} J \triangle K\right),
$$

so that by (3.2),

$$
\mu_{*}\left(A^{c} \cap K\right) \leq \mu_{*}\left(A^{c} \cap z^{-n} J\right)+\mu\left(z^{-n} J \triangle K\right) .
$$

Using this fact we now have

$$
\begin{aligned}
\mu_{*}\left(A^{c} \cap J\right) & =\mu_{*}\left(\rho_{z^{-n}}\left(A^{c} \cap J\right)\right), \quad \text { by }(3.4), \\
& =\mu_{*}\left(z^{-n}\left(A^{c} \cap J\right)\right) \\
& =\mu_{*}\left(z^{-n} A^{c} \cap z^{-n} J\right) \\
& =\mu_{*}\left(A^{c} \cap z^{-n} J\right) \quad \text { as } A^{c} \text { is } \rho_{z^{-} \text {-invariant }} \\
& \geq \mu_{*}\left(A^{c} \cap K\right)-\mu\left(z^{-n} J \triangle K\right), \quad \text { by }(4.5) \\
& \geq \mu_{*}\left(A^{c} \cap K\right)-\delta, \quad \text { by }(5.2), \\
& \geq(1-\varepsilon) \mu(K)-\delta, \quad \text { by }(5.1), \\
& =(1-\varepsilon) \mu(J)-\delta,
\end{aligned}
$$

as $\mu(J)=\mu(K)$, again using (5.1). Note that $A$ and $J$ are independent of $\delta$ and that (5.3) holds for all $\delta>0$. Hence,

$$
\mu_{*}\left(A^{c} \cap J\right) \geq(1-\varepsilon) \mu(J) .
$$

Now (5.1) and (5.4) give

$$
\mu_{*}(A \cap J)+\mu_{*}\left(A^{c} \cap J\right) \geq 2(1-\varepsilon) \mu(J) .
$$


Recall that $\varepsilon$ was any number such that $\varepsilon>0$, so that $2(1-\varepsilon)$ may be as close to 2 as specified in advance. So, summarizing the discussion in terms of (5.5) we have:

If $z$ is an element of $\mathbb{T}$ which is not a root of unity, and if $A$ is a $\rho_{z}$-invariant subset of $\mathbb{T}$ with the properties that $\mu_{*}(A)>0$ and $\mu_{*}\left(A^{c}\right)>0$; then for each positive number $\eta<2$ there is an $\operatorname{arc} J$ of $\mathbb{T}$, this arc depending upon $\eta$, such that

$$
\mu_{*}(A \cap J)+\mu_{*}\left(A^{c} \cap J\right) \geq \eta \mu(J) .
$$

The following result is simply a contrapositive version of (5.6).

Theorem 5.1 Let $z$ be an element of $\mathbb{T}$ which is not a root of unity, and let $A$ be $a$ $\rho_{z}$-invariant subset of $\mathbb{T}$. Suppose that there is $\theta<2$ such that for all arcs $J$ of $\mathbb{T}$,

$$
\mu_{*}(A \cap J)+\mu_{*}\left(A^{c} \cap J\right) \leq \theta \mu(J) .
$$

Then, either $A$ or $A^{c}$ is a set of outer measure zero.

Note that by (3.2), it is always the case that

$$
\mu(J)=\mu_{*}(J) \leq \mu_{*}(A \cap J)+\mu_{*}\left(A^{c} \cap J\right) \leq 2 \mu(J) .
$$

Hence, the "simplest" way in which condition (5.7) may be satisfied is to have

$$
\mu_{*}(A \cap J)+\mu_{*}\left(A^{c} \cap J\right)=\mu(J),
$$

for all $\operatorname{arcs} J$ of $\mathbb{T}$. However, if $A$ is any set for which this happens, it follows by [3, pp. 63-64] that

$$
\mu_{*}(A \cap B)+\mu_{*}\left(A^{c} \cap B\right)=\mu(B),
$$

for all subsets $B$ of $\mathbb{T}$, not just for arcs, so that $A$ must be measurable in this case. (Note that in [3, p. 63], equation (5.8) is taken as the definition of a measurable set.)

Thus, the problem of calculating the outer measure of an invariant set of an irrational rotation has led to condition (5.7). However, the simplest way in which this condition can be satisfied is for the set to satisfy the Carathéodory definition of a measurable set. So, in this sense, the problem of calculating the outer measure of an invariant set of an irrational rotation has motivated the definition of the notion of a measurable set.

Now, if $A$ is a $\rho_{z}$-invariant set which satisfies (5.7), Theorem 5.1 implies that either $A$ or $A^{c}$ is a set of outer measure zero. By the remarks in Section 3 , it then follows that $A$ must be measurable. Thus, a $\rho_{z}$-invariant set $A$ which satisfies (5.7) must be measurable, so that in fact it satisfies condition (5.9) which is formally stronger than (5.7).

Theorem 5.1 leaves open the question as to whether, for some given irrational rotation $\rho$, there exists a $\rho$-invariant, non-measurable set. However, for any irrational rotation $\rho$, in a personal communication Peter Nickolas has given an example of a set $A$ which is $\rho$-invariant and non-measurable. The preceding remarks show that such a set will have the property that $\mu_{*}(A)>0$ and $\mu_{*}\left(A^{c}\right)>0$. Thus the conjecture at the end of Section 3 is false. Theorem 5.1 can be regarded as a refinement of the conjecture which happens to be true.

Any non-measurable set $A$ which is also invariant for some irrational rotation must have the property that $\mu_{*}(A)=\mu_{*}\left(A^{c}\right)=1$, as shown in the following result. 
Theorem 5.2 Let $z$ be an element of $\mathbb{T}$ which is not a root of unity, and let $A$ be a subset of $\mathbb{T}$ which is $\rho_{z}$-invariant. Then, if $A$ is non-measurable, $\mu_{*}(A)=\mu_{*}\left(A^{c}\right)=1$.

Idea of the proof. If $A$ is not measurable, the remarks above show that $A$ does not satisfy (5.7). Hence, for each $1<\eta<2$, there is an arc $J$ such that

$$
\mu_{*}(A \cap J)+\mu_{*}\left(A^{c} \cap J\right) \geq \eta \mu(J),
$$

as in (5.6). By an argument similar to that in Lemma 4.2, we can show that this arc $J$ may have a length as small as we please. Then, if $K$ is any arc of the form $\rho_{z}^{[n]}(J),(5.6)$ will hold with $K$ in place of $J$, because $A$ is $\rho_{z}$-invariant and the outer measure does not change under rotations. By taking a suitable finite disjoint union of such $\operatorname{arcs} K$, we get a set $B$ such that $\mu(B)>\eta-1$ and

$$
\mu_{*}(A \cap B)+\mu_{*}\left(A^{c} \cap B\right) \geq \eta \mu(B) .
$$

We deduce that

$$
\mu_{*}(A)+\mu_{*}\left(A^{c}\right) \geq \mu_{*}(A \cap B)+\mu_{*}\left(A^{c} \cap B\right) \geq \eta \mu(B) \geq \eta(\eta-1) .
$$

Since this is true for all $1<\eta<2$, it follows that

$$
\mu_{*}(A)+\mu_{*}\left(A^{c}\right)=2 .
$$

Since $\mu_{*}(A) \leq 1$ and $\mu_{*}\left(A^{c}\right) \leq 1$, this gives $\mu_{*}(A)=\mu_{*}(A)=1$.

The standard way of looking at the invariant sets of an irrational rotation is to restrict attention to the measurable sets. Note that when the set $A$ is measurable, it is usual to denote the outer measure $\mu_{*}(A)$ of $A$ simply by $\mu(A)$.

Theorem 5.3 Let $z$ be an element of $\mathbb{T}$ which is not a root of unity, and let $A$ be $a$ measurable subset of $\mathbb{T}$ which is $\rho_{z}$-invariant. Then, either $\mu(A)=0$ or $\mu(A)=1$.

Proof. Theorem 5.1 shows that $\mu(A)=0$ or $\mu\left(A^{c}\right)=0$. If $\mu(A)=0$, there is nothing to prove; while if $\mu\left(A^{c}\right)=0$, the additivity of $\mu$ on the measurable sets gives

$$
\mu(A)=\mu(\mathbb{T})-\mu\left(A^{\mathcal{c}}\right)=1-0=1 .
$$

Theorem 5.3 is the standard way of stating that an irrational rotation on the unit circle is ergodic (see [13, p. 27, 29]), and assumes the knowledge of what a measurable set is and the knowledge that the outer measure is additive when it is restricted to the measurable sets. Theorem 5.1 can be viewed as the result which can be obtained in place of Theorem 5.3 when one does not assume any knowledge of measurable sets, nor any knowledge of the additivity of the outer measure on the measurable sets. The present proof of Lemma 4.1 should be compared with the proof given by Sinai in [12, pp. 39-40], mentioned earlier. The notion of an arc of density can be regarded as a crude notion of a point of density, and the proof here may be regarded as a cruder approach than Sinai's to the problem of the ergodic behaviour of an irrational rotation. But it is precisely the use of these cruder tools which has led to the additional information in Theorem 5.1 and to the materializing of the Carathéodory definition of a measurable set. 


\section{Conclusions}

If $\rho$ is an irrational rotation on $\mathbb{T}$, a subset $A$ of $\mathbb{T}$ is called almost invariant if $\mu_{*}(A \triangle \rho(A))=0$. Of course, if $A$ happens to be $\rho$-invariant, $A \triangle \rho(A)=A \triangle A=\emptyset$, so that an invariant set is almost invariant. Some treatments of ergodic transformations are based on almost invariant sets rather than invariant sets, and it is known that the two approaches are equivalent in the usual setting. That is, a transformation is ergodic in the sense that every measurable invariant set has measure 0 or 1 if and only if the transformation is ergodic in the sense that every measurable almost invariant set has measure 0 or 1 [13, p. 27]. The same equivalence obtains in the present context, in that the equivalences in Theorem 5.1 remains true if the assumption that $A$ is invariant is replaced by the assumption that $A$ is almost invariant. Essentially, this is because if $A$, $B$ are two subsets of $\mathbb{T}$ such that $\mu_{*}(A \triangle B)=0$, then $\mu_{*}(A)=\mu_{*}(B)$.

The approach to ergodicity, discussed here for rotations, has been carried out in terms of the outer measure only. This approach may also be carried out in some other contexts. For example, let $\xi$ be the function on $\mathbb{T}$ given by $z \longmapsto z^{2}$. Then the following result is a standard one for measurable sets (see [13, p. 29] for a proof), but the proof in [9] gives a more general result while at the same time being more elementary.

Theorem 6.1 If $A$ is a subset of $\mathbb{T}$ which is $\xi$-invariant, then $\mu_{*}(A)=0$ or $\mu_{*}(A)=1$.

Note that if the $\xi$-invariant set $A$ in Theorem 6.1 is non-measurable, then $A^{c}$ is also non-measurable. Then, because any set of outer measure zero is measurable it follows that, in this case, $\mu_{*}(A)=\mu_{*}\left(A^{c}\right)=1$.

It is interesting to note the difference in behaviour between an irrational rotation $\rho$ and the function $\xi$. Whereas the conclusion in Theorem 5.1 that $\mu_{*}(A)=0$ or $\mu_{*}\left(A^{c}\right)=0$ depends upon some assumption about the $\rho_{z}$-invariant subset $A$ which goes beyond the mere fact that it is a subset, the conclusion in Theorem 6.1 that $\mu_{*}(A)=0$ or $\mu_{*}(A)=1$ requires no particular assumption about the $\xi$-invariant set $A$. This difference can be seen as arising from the facts that $\rho$ "preserves" the distance between points, but $\xi$ "stretches" the distance between points. Similar differences are seen also in other contexts. For example, $\rho$ is not weak mixing but $\xi$ is strong mixing [13, p. 50]. Also, in [10] it is pointed out that when $[0,1)$ is identified with $\mathbb{T}$ under the map $t \longmapsto \exp (2 \pi i t)$, and when $\rho$ is then regarded as a (discontinuous) function on $[0,1)$ instead of $\mathbb{T}$, then $\rho$ has a "weak" form of chaotic behaviour compared with more familiar examples of chaos, such as those exemplified by the function $\xi$ when it is also regarded as a function on $[0,1)$. These differences in behaviour are also related to the one-to-one nature of $\rho$, while $\xi$ is not one-to-one.

Acknowledgements. I am indebted to Michael Cwikel, Peter Nickolas and Graham Williams for many helpful comments. In particular, I am indebted to Peter for his example of a non-measurable invariant set for a given irrational rotation. 


\section{References}

[1] Bartle, R.: The Elements of Integration, Wiley 1966.

[2] Carathéodory, C.: Über das lineare Maß von Punktmengen: eine Verallgemeinerung des Längenbegriffs, Nachr. Gesell. Wiss. Göttingen (1914), 404-426.

[3] Craven, B.D.: Lebesgue Measure and Integral, Pitman 1982.

[4] Halmos, P.: Measure Theory, van Nostrand 1965.

[5] Hersh, R.: What is Mathematics, Really?, Vintage 1998.

[6] Hewitt, E., and Stromberg, K.: Real and Abstract Analysis, Springer-Verlag 1965.

[7] Lakatos, I.: Proofs and Refutations; the logic of mathematical discovery, (Worrall, J., and Zahar, E., eds.), Cambridge University Press 1976.

[8] Loève, M.: Probability Theory, van Nostrand 1955.

[9] Nillsen, R.: Statistical Behaviour in Dynamical Systems, School of Mathematics and Applied Statistics, University of Wollongong 1998.

[10] Nillsen, R.: Chaos and one-to-oneness, Mathematics Magazine, 72 (1999), 14-21.

[11] Rudin, W.: Real and Complex Analysis, McGraw-Hill 1966.

[12] Sinai, Y.G.: Introduction to Ergodic Theory, translated by V. Scheffer, Princeton University Press 1977.

[13] Walters, P.: An Introduction to Ergodic Theory, Springer-Verlag 1982.

Rodney Nillsen

School of Mathematics and Applied Statistics

University of Wollongong

Northfields Avenue

Wollongong, NSW 2522, Australia

e-mail: rodney_nillsen@uow.edu.au 